\title{
Sensitivity Minimization by Stable Controllers for a Class of Unstable Time-Delay Systems
}

\author{
Suat Gümüşsoy \\ was with Dept. of Electrical and Computer Eng., \\ Ohio State University, Columbus, OH 43210, U.S.A. \\ current affiliation: MIKES Inc., Akyurt, \\ Ankara, TR-06750, Turkey \\ e-mail: suat.gumussoy@mikes.com.tr
}

\author{
Hitay Özbay \\ Dept. of Electrical and Electronics Eng., \\ Bilkent University, Bilkent, Ankara TR-06800, Turkey, \\ on leave from Dept. of Electrical and Computer Eng., \\ Ohio State University, Columbus, OH 43210, U.S.A. \\ e-mail: hitay@bilkent.edu.tr
}

\begin{abstract}
In this paper sensitivity minimization problem is considered for a class of unstable time delay systems. Our goal is to find a stable controller stabilizing the feedback system and giving rise to smallest $\mathcal{H}^{\infty}$ norm for the sensitivity function. This problem has been solved by Ganesh and Pearson (1986) for finite dimensional plants using Nevanlinna-Pick interpolation. We extend their technique to include possibly unstable time delay systems. Moreover, we illustrate suboptimal solutions, and their robust implementation.
\end{abstract}

Keywords-strong stabilization, time-delay, sensitivity minimization, H-infinity control

\section{INTRODUCTION}

In feedback control applications, sometimes it is desirable to have a stable controller which internally stabilizes the closedloop. There are many practical reasons why we want the controller itself to be stable, [19]. A necessary and sufficient condition for the existence of a stable controller stabilizing the feedback system for a given plant is the parity interlacing property, [20]. Design of such controllers is known as strong stabilization problem and several methods are available for its solution for MIMO or SISO finite dimensional plants, [2$5,9,11,12,14-17,22,23]$ as well as different classes of SISO time delay systems, [8], [18], under $\mathcal{H}^{\infty}, \mathcal{H}_{2}$ or other optimization constraints. Notably, the design methods in [1], [7] give optimal stable $\mathcal{H}^{\infty}$ controllers for finite dimensional SISO plants as a solution to weighted sensitivity minimization problem, other methods provide sufficient conditions to find stable $\mathcal{H}^{\infty}$ controllers.

In this paper, the method of [7] is generalized for a class of time-delay systems. The plants we consider may have infinitely many right half plane poles. Optimal and suboptimal stable $\mathcal{H}^{\infty}$ controllers are obtained for the weighted sensitivity minimization problem using the Nevanlinna-Pick interpolation.

In section [1] the control problem is defined and the structure of the plant is given. In section III we summarize our earlier results on the necessary and sufficient conditions to write the plant in the given structure for a class of possibly unstable time-delay systems. Main results are given in section IV An example can be found in section $\mathrm{V}$, and concluding remarks are made in last section.

\section{Problem Definition}

Given a single-input-single-output linear time invariant plant $P$, sensitivity function of the feedback system is defined as $S:=(1+P C)^{-1}$, where $C$ is the controller to be designed. We say that the feedback system is stable if $S, P S, C S$ are stable transfer functions (i.e. they are in $\mathcal{H}^{\infty}$ ). Moreover, if a stable controller, $C \in \mathcal{H}^{\infty}$, stabilizes the feedback system, then $C$ is said to be strongly stabilizing, [19]. For a given plant $P$, the set of all strongly stabilizing controllers is denoted by $\mathcal{S}_{\infty}(P)$.

For a given minimum phase function $W(s)$, the problem of weighted sensitivity minimization by stable controller (WSMSC) is to find

$$
\begin{aligned}
\gamma_{s} & =\inf _{C \in \mathcal{S}_{\infty}(P)}\left\|W(1+P C)^{-1}\right\|_{\infty}, \\
& =\left\|W\left(1+P C_{\gamma_{s}}\right)^{-1}\right\|_{\infty}
\end{aligned}
$$

where $\gamma_{s}$ is the minimum $\mathcal{H}^{\infty}$ cost for WSMSC and $C_{\gamma_{s}} \in$ $\mathcal{S}_{\infty}(P)$ is the corresponding optimal strongly stabilizing controller.

We assume that the transfer function of the plant can be factored as

$$
P(s)=\frac{m_{n}(s)}{m_{d}(s)} N_{o}(s)
$$

where $m_{d}, m_{n}$ are inner (all-pass) functions, $m_{n}$ is finite dimensional and $m_{d}$ is infinite dimensional; $N_{o}$ is outer (minimum phase) and possibly infinite dimensional.

In section IV we will obtain the optimal controller $C_{\gamma_{s}} \in$ $\mathcal{S}_{\infty}(P)$ for the WSMSC problem, where the plant $P$ admits a factorization of the form (3). But first, in the next section, we shall illustrate how this factorization can be done for a class of possibly unstable systems with time delays.

\section{Plant Factorization for Time Delay Systems}

In this section, we summarize some preliminary results from [10] on the factorization of SISO time-delay systems in the form (3).

The plants we consider in this paper are assumed to be in the form

$$
P(s)=\frac{R(s)}{T(s)}=\frac{\sum_{i=1}^{n} R_{i}(s) e^{-h_{i} s}}{\sum_{j=1}^{m} T_{j}(s) e^{-\tau_{j} s}}
$$


where $R_{i}$ and $T_{j}$ are finite dimensional, stable, proper transfer functions, and time delays $h_{i}, \tau_{j}$ are assumed to be positive rational numbers, with $0 \leq h_{1}<\ldots<h_{n}$ and $0 \leq \tau_{1}<$ $\ldots<\tau_{m}$.

Definition 3.1: Consider $R(s)=\sum_{i=1}^{n} R_{i}(s) e^{-h_{i} s}$ as defined above. Let $d_{i}$ be the relative degree of $R_{i}(s)$. Then,

1) if $d_{1}<\max \left\{d_{2}, \ldots, d_{n}\right\}, R(s)$ is called as retardedtype time-delay system (RTDS),

2) if $d_{1}=\max \left\{d_{2}, \ldots, d_{n}\right\}, R(s)$ is called as neutral-type time-delay system (NTDS),

3) if $d_{1}>\max \left\{d_{2}, \ldots, d_{n}\right\}, R(s)$ is called as advancedtype time-delay system (ATDS).

The following lemma gives a necessary and sufficient condition when a NTDS has finitely many unstable zeros.

Lemma 3.1: ([10]) Assume that $R(s)$ is a NTDS with no imaginary axis zeros and poles, then the system, $R$, has finitely many unstable zeros if and only if all the roots of the polynomial, $\varphi(r)=1+\sum_{i=2}^{n} \xi_{i} r^{\tilde{h}_{i}-\tilde{h}_{1}}$ has magnitude greater than 1 where

$$
\begin{aligned}
\xi_{i} & =\lim _{\omega \rightarrow \infty} R_{i}(j \omega) R_{1}^{-1}(j \omega) \quad \forall i=2, \ldots, n, \\
h_{i} & =\frac{\tilde{h}_{i}}{N}, \quad N, \quad \tilde{h}_{i} \in Z_{+}, \forall i=1, \ldots, n .
\end{aligned}
$$

By the following corollary, all SISO time-delay systems with finitely many unstable zeros are obtained.

Corollary 3.1: ([10]) The time-delay system $R$ has finitely many unstable zeros if and only if $R$ is a RTDS or $R$ is a NTDS satisfying Lemma 3.1 Time-delay systems with finitely many unstable zeros are defined as $F$-systems.

We define the conjugate of $R(s)=\sum_{i=1}^{n} R_{i}(s) e^{-h_{i} s}$ as $\bar{R}(s):=e^{-h_{n} s} R(-s) M_{C}(s)$ where $M_{C}$ is inner, finite dimensional whose poles are poles of $R$. The time-delay system $\bar{R}$ has finitely many unstable zeros if and only if $R$ is a ATDS or Lemma 3.1 is satisfied by $\bar{R}$. The time-delay system $R$ whose conjugate $\bar{R}$ has finitely many unstable zeros is defined as an $I$-system.

The class of SISO time-delay systems with factorization (3) is given by the following lemma.

Lemma 3.2: ([10]) If $R$ is an $F$ system and $T$ is an $I$ system in (4), then $P$ can be factored as (3). If $R$ and $T$ are irreducible and have no common factors, then $P$ has factorization (3) if and only if $R$ and $T$ are $F$ and $I$ system respectively.

In this paper, the plant $P$, defined by (4), is assumed to satisfy the following:

A.1 $R_{i}$ and $T_{j}$ are stable, proper, finite dimensional transfer functions. The delays, $h_{i}, \tau_{j}$ are rational numbers such that $0 \leq h_{1}<h_{2}<\ldots<h_{n}$, and $0 \leq \tau_{1}<\tau_{2}<\ldots<$ $\tau_{m}$, with $h_{1}=\tau_{1}=0$.

A.2 $R$ and $T$ have no imaginary axis zeros.

A.3 $R$ and $T$ are $F$ and $I$ system respectively.

Under the above conditions $P$ can be factored as in (3),

$$
m_{d}=M_{\bar{T}} \frac{T}{\bar{T}}, \quad m_{n}=M_{R}, \quad N_{o}=\frac{R}{M_{R}} \frac{M_{\bar{T}}}{\bar{T}} .
$$

The zeros of the inner function $M_{R}$ are right half plane zeros of $R$. The unstable zeros of $\bar{T}(s)$ are the same as the zeros of the inner function $M_{\bar{T}}$. The conjugate of $T$ has finitely many unstable zeros since $T$ is a $I$-system.

As an example, consider the following time-delay system:

$$
\begin{aligned}
& \dot{x}(t)=-x(t)-2 \dot{x}(t-2)+2 x(t-2)+u(t), \\
& y(t)=4 x(t-3)-2 \dot{x}(t-2)+2 x(t-2)+u(t)
\end{aligned}
$$

which has the transfer function

$$
P(s)=\frac{(s+1)+4 e^{-3 s}}{(s+1)+2(s-1) e^{-2 s}} .
$$

The plant $P$ can be written in the form of (4),

$$
\begin{aligned}
P & =\frac{R}{T}=\frac{R_{1} e^{-h_{1} s}+R_{2} e^{-h_{2} s}}{T_{1} e^{-\tau_{1} s}+T_{2} e^{-\tau_{2} s}}, \\
& =\frac{1 e^{-0 s}+\left(\frac{4}{s+1}\right) e^{-3 s}}{1 e^{-0 s}+\left(\frac{2(s-1)}{s+1}\right) e^{-2 s}} .
\end{aligned}
$$

Note that $P$ satisfies assumption A.1 (i.e., $h_{1}=\tau_{1}=0$ ) and A.2 since it has no imaginary axis zeros and poles. The relative degree of $R_{2}$ is larger than $R_{1}$, therefore, $R$ is a RTDS and has finitely many unstable zeros (it is an $F$ system). The conjugate of $T$ is

$$
\begin{aligned}
\bar{T}(s) & =e^{-2 s} T(-s)\left(\frac{s-1}{s+1}\right), \\
& =2+\left(\frac{s-1}{s+1}\right) e^{-2 s} .
\end{aligned}
$$

Note that $\bar{T}$ is NTDS which satisfies Lemma 3.1. So, $\bar{T}$ has finitely many zeros and hence $T$ is an $I$ system. Therefore, the plant $P$ satisfies assumption A.3. It can be shown that $R$ has two unstable zeros at $s_{R_{1,2}}=0.3125 \pm 0.8548 j$. Also, $T$ has infinitely many unstable poles converging to $\ln \sqrt{2} \pm$ $j\left(k+\frac{1}{2}\right) \pi$ as $k \rightarrow \infty$, which shows that the plant $P$ has finitely many unstable zeros and infinitely many unstable poles. By the small-gain theorem, it is clear that $\bar{T}$ has no unstable zeros. Now $P$ can be written as in (3) where

$$
\begin{aligned}
& m_{d}(s)=\frac{T(s)}{\bar{T}(s)}, \\
& m_{n}(s)=M_{R}(s)=\frac{s^{2}-0.6250 s+0.8283}{s^{2}+0.6250 s+0.8283}, \\
& N_{o}(s)=\frac{R(s)}{M_{R}(s)} \frac{1}{\bar{T}(s)} .
\end{aligned}
$$

Note that $M_{R}$ is an inner function and all its zeros are unstable zeros of $R$. Since $\bar{T}$ has no unstable zeros, $M_{\bar{T}}$ is equal to one.

In the next section, stable $\mathcal{H}^{\infty}$ controllers are obtained for plants in the form (3).

\section{Stable $\mathcal{H}^{\infty}$ Controller Design}

In this section, the results of [7] are extended for plants with infinitely many unstable modes. The internal stability problem of closed-loop system can be reduced to interpolation problem on the sensitivity function [20]. This reduction is valid also for plants with infinitely many unstable poles and 
zeros. Assume that $P(s)=\frac{m_{n}(s)}{m_{d}(s)} N_{o}(s)$ is as defined above with finite dimensional inner $m_{n}$, infinite dimensional inner $m_{d}$, outer $N_{o}$. Note that the plant has finitely many unstable zeros and may have infinitely unstable poles. Let the weighting function, $W$, be minimum phase, then the closed-loop system is internally stable if and only if there exists $S_{W} \in \mathcal{H}^{\infty}$, $S_{W}=W(1+P C)^{-1}$ satisfying

$$
S_{W}(s)=m_{d}(s) F_{\gamma}(s)
$$

where $F_{\gamma} \in \mathcal{H}^{\infty}$, and

$$
m_{d}\left(s_{i}\right) F_{\gamma}\left(s_{i}\right)=W\left(s_{i}\right)
$$

for all zeros of $m_{n}(s), s_{i} \in \mathbb{C}_{+}, i=1 \ldots, N$. Moreover, $\left\|S_{W}\right\|_{\infty}=\left\|F_{\gamma}\right\|_{\infty}$. Optimal weighted sensitivity is the one which corresponds to an $F_{\gamma}$ whose $\mathcal{H}^{\infty}$ norm is the smallest among all stable functions satisfying (9).

When the controller in the weighted sensitivity minimization problem defined above is restricted to be stable, then we must have

$$
\begin{aligned}
C_{\gamma} & =\frac{W-S_{W}}{S_{W} P}=\frac{\left(W-\gamma m_{d} F\right) N_{o}^{-1}}{\gamma m_{d} F P} \\
& =\frac{\left(W-\gamma m_{d} F\right) N_{o}^{-1}}{\gamma m_{n} F} \in \mathcal{H}^{\infty}
\end{aligned}
$$

where $F \in \mathcal{H}^{\infty}$ and $F^{-1} \in \mathcal{H}^{\infty}$ with $\|F\|_{\infty} \leq 1$ and it satisfies the interpolation conditions

$$
F\left(s_{i}\right)=\frac{W\left(s_{i}\right)}{\gamma m_{d}\left(s_{i}\right)}=\frac{\omega_{i}}{\gamma}, \quad i=1, \ldots, N
$$

for the smallest possible $\gamma>0$. Conversely, if there exists such an $F$, then optimal stable $\mathcal{H}^{\infty}$ controller $C_{\gamma_{s}}$ for WSMSC problem (1) can be obtained from $S_{W}$. The optimal $\mathcal{H}^{\infty}$ cost for (2) is $\gamma_{s}$, which is the smallest $\gamma$ value for which a unit $F \in \mathcal{H}^{\infty}$ satisfying (10) can be found. (We say that a function $F \in \mathcal{H}^{\infty}$ is a unit if $F^{-1} \in \mathcal{H}^{\infty}$ and $\|F\|_{\infty} \leq 1$ ). Note that the above transformation reduces the WSMSC problem for plants with infinitely unstable modes into an interpolation problem, by a unit in $\mathcal{H}^{\infty}$, with finitely many interpolation conditions.

The solution of the interpolation problem with unit is given in [7] using the Nevanlinna-Pick approach, [6,13,21], as follows. Define

$$
G(s)=-\ln F(s) \quad F(s)=e^{-G(s)} .
$$

Now, we want to find an analytic function $G: \mathbb{C}_{+} \rightarrow \mathbb{C}_{+}$ such that

$$
G\left(s_{i}\right)=-\ln \omega_{i}+\ln \gamma-j 2 \pi m_{i}=: \nu_{i}, \quad i=1, \ldots, N
$$

where $m_{i}$ is a free integer due to non-uniqueness of the complex logarithm. Note that when $\|F\|_{\infty} \leq 1$ the function $G$ has a positive real part hence it maps $\mathbb{C}_{+}$into $\mathbb{C}_{+}$. Now if the extended right-half plane is transformed onto the closed unit disc in the complex plane by one-to-one conformal mapping $z=\phi(s)$, then the transformed interpolation conditions are

$$
f\left(z_{i}\right)=\frac{\omega_{i}}{\gamma}, \quad i=1, \ldots, N
$$

where $z_{i}=\phi\left(s_{i}\right)$ and $f(z)=F\left(\phi^{-1}(z)\right)$. The transformed interpolation problem is to find a unit with $\|f\|_{\infty} \leq 1$ such that interpolation conditions (12) are satisfied. By the following transformation,

$$
g(z)=-\ln f(z)
$$

the interpolation problem can be written as,

$$
g\left(z_{i}\right)=\nu_{i}, \quad i=1, \ldots, N
$$

Define $\phi\left(\nu_{i}\right)=: \zeta_{i}$. If we can find an analytic function $\tilde{g}$ mapping unit disc onto unit disc, satisfying

$$
\tilde{g}\left(z_{i}\right)=\zeta_{i} \quad i=1, \ldots, N
$$

then the desired $g(z)$, hence $f(z)$ and $F(s)$ can be constructed from $g(z)=\phi^{-1}(\tilde{g}(z))$. The problem of finding such $\tilde{g}$ is the well-known Nevanlinna-Pick problem, [6,13,21]. The condition for the existence of an appropriate $g$ can be given directly: there exists an analytic $g$ mapping the unit disc onto right half plane if and only if the Pick matrix $P_{N \times N}$,

$$
P\left(\gamma,\left\{m_{i}\right\}\right)_{i, k}=\left[\frac{2 \ln \gamma-\ln \omega_{i}-\ln \bar{w}_{k}+j 2 \pi m_{k, i}}{1-z_{i} \bar{z}_{k}}\right]
$$

is positive semi-definite, where $m_{k, i}=m_{k}-m_{i}$ are integers. In [7], it is mentioned that the possible integer sets $\left\{m_{i}\right\}$ are finite and in all possible integer sets $\left\{m_{i}\right\}_{l}, l=1, \ldots, r$, there exists a minimum value, $\gamma_{s}$, such that $P\left(\gamma_{s},\left\{m_{i}\right\}_{l}\right) \geq 0$.

\section{A. Optimal Stable $\mathcal{H}^{\infty}$ Controller Design Algorithm for Plants with Infinitely Unstable Modes:}

1) Write the plant in the form of (3):

If the plant is a SISO time-delay system, obtain its transfer function and re-write it in the form of (4). If $R$ and $T$ satisfy Assumptions A.1-A.3, do the factorization of the plant as (3).

2) Find the zeros $s_{i} i=1, \ldots, N$ of $m_{n}(s)$.

3) Calculate $\omega_{i}$, and using a conformal mapping $\phi$ calculate $z_{i}$ for $i=1, \ldots, N$.

4) For all possible integer sets $\left\{m_{i}\right\}_{l}, l=1, \ldots, r$, find $\gamma_{s}$ such that the Pick matrix (14) is positive semi-definite.

5) Obtain optimal interpolation function $g_{\gamma_{s}}(z)$ and $f_{\gamma_{s}}(z)$ by transformation [13, see e.g. [6,21].

6) Calculate $F_{\gamma_{s}}(s)=f_{\gamma_{s}}(\phi(s))$ and $S_{W, \gamma_{s}}(s)$ using $F_{\gamma_{s}}$ and $\gamma_{s}$ in 8 .

7) The optimal stable $\mathcal{H}^{\infty}$ controller for plants with infinitely unstable modes can now be written as

$$
C_{\gamma_{s}}=\frac{W-S_{W, \gamma_{s}}}{S_{W, \gamma_{s}} P}
$$

Note that this controller achieves the optimal $\mathcal{H}^{\infty}$ norm $\gamma_{s}$ which is the minimum value for WSMSC problem. 


\section{B. Remarks:}

1) Clearly, stable $\mathcal{H}^{\infty}$ controller design is also applicable to infinite dimensional plants with finitely many right half plane poles and zeros. In this case it is possible to write the plant as

$$
P(s)=\frac{m_{n}(s)}{m_{d}(s)} N_{o}(s)
$$

where $m_{n}$ and $m_{d}$ are finite dimensional inner functions whose zeros are $\mathbb{C}_{+}$zeros and poles of plant $P$ respectively; $N_{o}$ is outer, i.e. the minimum phase part of the plant $P$. For timedelay systems (4), this case means that $R$ and $T$ are $F$ systems. Stable $\mathcal{H}^{\infty}$ controller design for plants 15 is the same as in [7]. The main difference is that the term $m_{d}$ in $S_{W}$ is finite dimensional. There are many plants with the above structure, such as,

$$
\begin{aligned}
\dot{x}(t) & =\sum_{i=0}^{n_{A}} A_{i} x\left(t-h_{A, i}\right)+B u(t), \\
y(t) & =C x(t)+d u(t)
\end{aligned}
$$

where $A_{i} \in \mathbb{R}^{n \times n}, i=1, \ldots, n_{A}$ and $B, C, d$ are real valued vectors of appropriate dimensions. The state vector has dimension is $x(t):=\left[x_{1}(t), \ldots, x_{n}(t)\right]^{T}$ and the time-delays satisfy

$$
0 \leq h_{A, 1}<\ldots<h_{A, i}<\ldots<h_{A, n_{A}} .
$$

Optimal stable $\mathcal{H}^{\infty}$ controller can be found for the plant (16).

2) Note that optimal stable $\mathcal{H}^{\infty}$ controller is unique and it is not rational. For practical purposes, rational approximation of the optimal controller can be done with desired error bound or a rational controller can be searched in the set of suboptimal controllers determined from the suboptimal solutions of the Nevanlinna-Pick problem.

3) There are always unstable pole-zero cancellations in the controller terms, $\frac{W-S_{W, \gamma_{s}}}{m_{n}}$ and $N_{o}$ from interpolation conditions and factorization respectively. It is not possible to directly cancel the unstable pole-zeros since the optimal interpolating function $F$ in $S_{W, \gamma_{s}}$ is irrational. If the suboptimal controllers are considered, the interpolating function $F$ can be chosen as finite dimensional. Exact cancellations are possible for infinite dimensional plants (15) with finite dimensional $F$ in the term $\frac{W-S_{W, \gamma_{s}}}{m_{n}}$. If $F$ is finite dimensional and the plant is a time-delay system with factorization (3), the controller can be written in a form such that the controller has a finite impulse response structure which eliminates unstable polezero cancellation problem in $\frac{W-S_{W, \gamma_{s}}}{m_{n}}$ and $N_{o}$, see [10]. This new structure of controller makes possible to implement the controller practically. The example shows this structure in $\mathrm{V}$.

\section{EXAMPLE}

Optimal stable $\mathcal{H}^{\infty}$ controller for WSMSC problem is designed for time-delay plant (5) with weighting function $W(s)=\frac{1+0.1 s}{s+1}$. The time-delay system (5) is put in the form of (7). The zeros of plants are $s_{R_{1,2}}=0.31 \pm 0.85 j$, and $\omega_{1,2}=0.79 \mp 0.42 j$. This gives the optimal $\mathcal{H}^{\infty}$ cost $\gamma_{s}=1.07$.
The algorithm gives the optimal $\mathcal{H}^{\infty}$ cost for WSMSC problem, that is the best value for any stable controller. Unfortunately, the resulting optimal stable $\mathcal{H}^{\infty}$ controller has internal unstable pole-zero cancellations. If the suboptimal case is considered, a practical controller can be found.

Consider a suboptimal solution to WSMSC for $\gamma=1.5$ which is larger than the optimal cost, $\gamma_{s}=1.07$. By a numerical search algorithm, a finite dimensional interpolating function $F_{\text {subopt }}$ can be found as

$$
F_{\text {subopt }}(s)=\frac{0.1895 s+0.7308}{s+0.7310} .
$$

Note that $F_{\text {subopt }}$ is a unit with $\left\|F_{\text {subopt }}\right\|_{\infty} \leq 1$ and satisfies the interpolation conditions $F_{\text {subopt }}\left(s_{R_{i}}\right)=\gamma^{-1} \omega_{i}$ for $i=$ 1,2 . The corresponding suboptimal sensitivity function can be obtained as $S_{W, \gamma}=\gamma m_{d} F_{\text {subopt }}$. The suboptimal stable $\mathcal{H}^{\infty}$ controller is

$$
\begin{aligned}
C_{\gamma} & =\frac{W-S_{W, \gamma}}{S_{W, \gamma} P}, \\
& =\left(\frac{\gamma^{-1} W F_{\text {subopt }}^{-1}-m_{d}}{m_{n}}\right) \frac{1}{N_{o}} .
\end{aligned}
$$

Note that there are unstable pole-zero cancellations inside the parenthesis in the above expression, and in $N_{o}$. It is clear that when the infinite dimensional plant admits a factorization (15), exact cancellation inside the parenthesis is possible because all the terms are finite dimensional. However, the plant in this example has an infinite dimensional part, $m_{d}$, so it is not possible to make exact cancellations in the controller. Nevertheless, the unstable pole-zero cancellations can be avoided by the method proposed in [10] as follows:

$$
\begin{aligned}
C_{\gamma} & =\left(\frac{\gamma^{-1} W F^{-1} \bar{T}-T}{m_{n}}\right)\left(\frac{R}{m_{n}}\right)^{-1}, \\
& =\left(H_{T}+\mathcal{F}_{T}\right)\left(H_{R}+\mathcal{F}_{R}\right)^{-1}
\end{aligned}
$$

where $\mathcal{F}_{T}$ and $\mathcal{F}_{R}$ are finite impulse response filters (i.e. their impulse responses are non-zero only on a finite time interval)

$$
\begin{aligned}
& \mathcal{F}_{R}(s)=\frac{1.25 s+(2.04 s+1.69) e^{-3 s}}{s^{2}-0.625 s+0.828} \\
& \mathcal{F}_{T}(s)=\frac{0.585 s+0.019-(0.285 s-1.066) e^{-2 s}}{s^{2}-0.625 s+0.828},
\end{aligned}
$$

whose denominators are determined from the zeros of $m_{n}$. The impulse responses of $\mathcal{F}_{T}$ and $\mathcal{F}_{R}$ are given in Figure 1 The terms, $H_{R}$ and $H_{T}$, are time-delay systems with no unstable pole-zero cancellations internally.

Note that if the plant has factorization $(15), \mathcal{F}_{T}=0$ since $m_{d}$ is finite dimensional. The exact cancellations can be made in $\frac{W-S_{W, \gamma}}{m_{n}}$ and $F_{R}$ is from unstable pole-zero cancellations inside $N_{o}$.

\section{CONCLUding REMARKS}

Weighted sensitivity minimization problem by stable $\mathcal{H}^{\infty}$ controllers is considered for SISO infinite dimensional plants with finitely many right half plane zeros and possibly infinitely many right half plane poles. The optimal stable $\mathcal{H}^{\infty}$ controller 

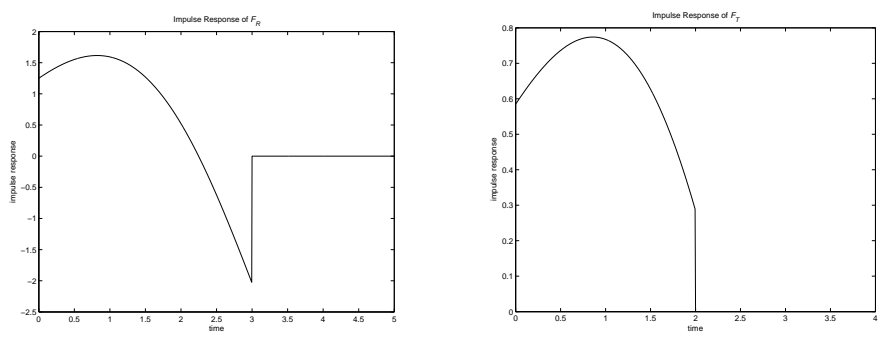

Fig. 1. Impulse Responses of $\mathcal{F}_{R}$ and $\mathcal{F}_{T}$

and corresponding optimal $\mathcal{H}^{\infty}$ cost are obtained from the Nevanlinna-Pick interpolation. For this purpose the approach of [7] is extended to the class of unstable time delay systems considered here. Suboptimal controllers can be found from all suboptimal interpolants determined by the Nevanlinna-Pick solutions, and infinite dimensional suboptimal controllers can be approximated by finite dimensional ones. It should be noted that when the plant has infinitely many right half plane zeros, there will be infinitely many interpolation conditions, and this approach will not be applicable in such cases. Another open problem in this area is the extension of the main results to a two-block $\mathcal{H}^{\infty}$ control problem, for example the mixed sensitivity minimization.

Acknowledgements: This work was supported in part by the European Commission (contract no. MIRG-CT-2004-006666) and by TÜBİTAK (grant nos. EEEAG-105E065 and EEEAG105E156).

\section{REFERENCES}

[1] A. E. Barabanov, "Design of $\mathcal{H}^{\infty}$ optimal stable controller," Proc. Conference on Decision and Control, pp. 734-738, 1996.

[2] D. U. Campos-Delgado and K. Zhou, " $\mathcal{H}^{\infty}$ Strong stabilization," IEEE Transactions on Automatic Control, vol.46, pp. 1968-1972, 2001.

[3] D. U. Campos-Delgado and K. Zhou, "A parametric optimization approach to $\mathcal{H}^{\infty}$ and $\mathcal{H}^{2}$ strong stabilizaiton," Automatica, vol. 39, No. 7, pp. 1205-1211, 2003.

[4] Y. Choi and W.K. Chung, "On the Stable $\mathcal{H}^{\infty}$ Controller Parameterization Under Sufficient Condition," IEEE Transactions on Automatic Control, vol.46, pp. 1618-1623, 2001.

[5] Y.S. Chou, T.Z. Wu and J.L. Leu, "On Strong Stabilization and $\mathcal{H}^{\infty}$ Strong-Stabilization Problems," Proc. Conference on Decision and Control, pp. 5155-5160, 2003.

[6] C. Foias, H. Özbay, A. Tannenbaum, Robust Control of Infinite Dimensional Systems: Frequency Domain Methods, Lecture Notes in Control and Information Sciences, No. 209, Springer-Verlag, London, 1996.

[7] C. Ganesh and J. B. Pearson, "Design of optimal control systems with stable feedback," Proc. American Control Conf., pp. 1969-1973, 1986.

[8] S. Gümüşsoy and H. Özbay, "On Stable $\mathcal{H}^{\infty}$ Controllers for Time-Delay Systems," in Proceedings of the 16th Mathematical Theory of Network and Systems, Leuven, Belgium, July 2004.

[9] S. Gümüssoy, and H. Özbay, "Remarks on Strong Stabilization and Stable $\mathcal{H}^{\infty}$ Controller Design," IEEE Trans. on Automatic Control, vol. 50, pp. 2083-2087, 2005.

[10] S. Gümüşsoy and H. Özbay, "Remarks on $\mathcal{H}^{\infty}$ Controller Design for SISO Plants with Time Delays," in the proceedings of the 5th IFAC Symposium on Robust Control Design, Toulouse, France, July, 2006.

[11] H. Ito, H. Ohmori and A. Sano, "Design of stable controllers attaining low $\mathcal{H}^{\infty}$ weighted sensitivity," IEEE Transactions on Automatic Control, vol.38, pp. 485-488, 1993.

[12] M. Jacobus, M. Jamshidi, C. Abdullah, P. Dorato and D. Bernstein, "Suboptimal strong stabilization using fixed-order dynamic compensation," Proc. American Control Conference, pp. 2659-2660, 1990.
[13] M. G. Krein and A. A. Nudel'man The Markov Moment Problem and Extremal Problems, Translations of Mathematical Monographs, Vol. 50, AMS, 1977.

[14] P.H. Lee and Y.C. Soh, "Synthesis of stable $\mathcal{H}^{\infty}$ controller via the chain scattering framework," System and Control Letters, vol.46, pp. 19681972, 2002.

[15] I. Petersen, "Robust $\mathcal{H}^{\infty}$ control of an uncertain system via a stable output feedback controller," Proc. American Control Conference, pp. 5000-5007, 2006.

[16] A.A. Saif, D. Gu and I. Postlethwaite, "Strong stabilization of MIMO systems via $\mathcal{H}^{\infty}$ optimization," System and Control Letters, vol.32, pp. 111-120, 1997.

[17] A. Sideris and M. G. Safonov, "Infinity-norm optimization with a stable controller," Proc. American Control Conference, pp. 804-805, 1985.

[18] K.Suyama, "Strong stabilization of systems with time-delays," Proc. IEEE Industrial Electronics Society Conference, pp. 1758-1763, 1991.

[19] M. Vidyasagar, Control System Synthesis: A Factorization Approach, MIT Press, 1985.

[20] D.C. Youla, J.J. Bongiorno and C.N. Lu, "Single-loop feedback stabilization of linear multivariable dynamical plants," Automatica, vol.10, pp. 159-173, 1974.

[21] M. Zeren and H. Özbay, "Comments on 'Solutions to Combined Sensitivity and Complementary Sensitivity Problem in Control Systems'," IEEE Transactions on Automatic Control, vol.43, p. 724, 1998.

[22] M. Zeren and H. Özbay, "On the synthesis of stable $\mathcal{H}^{\infty}$ controllers," IEEE Transactions on Automatic Control, vol.44, pp. 431-435, 1999.

[23] M. Zeren and H. Özbay, "On the strong stabilization and stable $H^{\infty}$ _ controller design problems for MIMO systems," Automatica, vol.36, pp. $1675-1684,2000$. 